\title{
Diagnosis Value of Membrane Glycolipids Biochemistry Index in Intracranial and Gastrointestinal Tumors
}

\author{
Jun Lv, Can-Qun Lv*, Ping Mei, Shi-Mei Qi
}

\begin{abstract}
The diagnostic value of membrane glycolipid biochemistry index, the lipid-bound sialic acid (LSA) and total sialic acid (TSA) in cerebrospinal fluid (CSF) was evaluated in 30 intracranial and 65 gastrointestinal tumors. The plasma LSA, TSA and red cell membrane sialic acid (R-SA) in were determined according to the method of Sevenmerhulm. Our results showed that the levels of LSA and TSA in CSF of intracranial tumor patients was higher than that of normal group $(p<0.01)$. The concentration of TSA and LSA in patients with malignant glioma was higher than that of benign meningioma patients $(P<0.01)$. No significance was found between intracranial halmatoma patients and normal control group for levels of membrane glycolipids $(p>0.05)$. Results also found that the plasma LSA, TSA and R-SA of gastric carcinoma were significantly higher than those of control group $(p<0.05)$; while no significant difference was found in the plasma LSA, TSA and R-SA levels between chronic gastritis, gastrohelcoma and normal control group $(p>0.05)$. Plasma LSA, TSA and R-SA levels of gastric carcinoma patient were significantly higher than those of chronic gastritis patients and gastrohelcoma patients $(p<0.05)$. It was also found that plasma LSA, TSA and R-SA contents were significantly higher in large intestine carcinoma patients than in benign in stestine tumor patients $(p<0.05)$ while no significant difference was found between intestine benign tumor and normal control group $(p>0.05)$. The levels of LSA, TSA and R-SA were obviously higher in the patients with metastasis than in the ones without $(p<0.05$.) The membrane glycolipid biochemistry index LSA and TSA in CSF are sensive markers for diagnosing intracranial tumors. For gastrointestinal malignant tumors the plasma LSA TSA and red blood cell membrane SA may be considered as auxiliary indicators for diagnosis. They can be used for distinguishing benign from malignant tumors.
\end{abstract}

Keywords: Membrane glycolipid - ganglioside - cerebrospinal fluid - intracranial tumor - gastroinstinal tumor

Asian Pac J Cancer Prev, 16 (7), 2693-2696

\section{Introduction}

Ganglioside (GIS) is important lipid constituents of cell membranes. Chemically gangliosides are glycosphingolipids. containg sialic acid (SA) (Lacomba et al., 2011) and are particulary abundant in membrane microdomains considered as highly organized units with various specific function (Sagawa et al., 1988; Gilbert et al., 2013). Gangliosides are involved in many significant cellular processes, such as cell growth, cell proliferation, differentiation and oncogenic transfrormation (Mansson et al., 1985; Yin et al., 2013). Animal brain tissue is highly enriched in ganglioside- phylogenetic and ontogenic differences in brain ganglioside biosynthesis has been evidenced as well as regional distribution and specific ganglioside patterns characterizing neuronal and glial cells during brain development, aging, and neurodegeneration. Specific change of gengliosides pattern in brain tumors reflecting tumor histoputhological origin, malignancy and progression has been extensively studied (Schiopu et al., 2009; Banda et al., 2012; Rathod et al., 2014) .

Little known about the value of dermination sialic acid in CSF of brain tumors, the aim of this study is to estimate the value of membrane glycolipid biochemistry index: plasma lipid-bound sialic acid (LSA), total sialic acid (TSA) and red blood cell membranes sialic acid (R-SA) in gastrointestial tumors: and to investigate the relationship between the above index and the distinguishment of benign tumors from malignant ones, tumor metastasis as well as prognosis judgement.

\section{Materials and Methods}

The clinical diagnosis and localigation for magligant glioma ( 12 cases), metastatic brain tumors ( 8 case), benign meningious (10 cases) and ntracr vanial halmatoma (8 cases) were determined using computerized tomography (CT) and magnetic resonance imaging (MRI). The diagnosis criteria for gastrointestinal tumors were followed by clinical diagnosis and by final pathohistological analysis of postoperative tumor tissue. The level of membrane glycolipid index (lipid-bound sialic acid (LSA) and total sialic acid (TSA) in CSF) was determined. The 65 patients with gastrointestinal malignant tumors 
also recruited in this study, which consist of 35 subjects with gastric carcinoma and 30 patients with colorectal carcinoma. Among the 65 case, 12 case with metastasis, 8 cases without metastasis. Informed consent was obtained, and the study protocol was approved by the Ethics Committee of Wannan Medical College. The contents of LSA, TSA and R-SA were determined based on the method of Sevennerholm progress improde, The genglioside extrcction and purification was performed, the extraction steps used chloroform (C), methanol (M) and water $(\mathrm{W})$ in a final volume ratio 1:1:0.8 (C: M:W) $(\mathrm{v} / \mathrm{v} / \mathrm{v})$, upper phases containing gangliosides were collected and air-dried, and obtained dry ganglioside extract was finally purified by dialysis and get-filtration. Purified ganglioside extract was used for quantitative analysis. The plasma LSA, TSA of cerebrospinal fluid (CSF) for intracranial tumors and the LSA, TSA and R-SA in gastrointestinal tumors was determined by resorcinol spectrometric method. The plasma was isolated from the whole blood by routine laboratory method, the plasma and red blood cells was finally separated by centrifugation. The red blood cells membrane was extracted from the red blood cell add water solve.

\section{Statistical analysis}

All statistical analyses were performed by SPSS, version13.0. Unpaired Student's t-test was used to compare the level of membrane glycolipids biochemistry index, the lipid-bound sialic acid (LSA) and total sialic acid (TSA) between two groups. The results were presented as the means $( \pm \mathrm{SD})$. A two-tailed $\mathrm{P}$ value of 0.05 was defined to be statistically significant.

\section{Results}

Table 1 shows that the levels of LSA in CSF of subjects with maligand glioma and benign menigions were higher than that of normal control group $(P<0.01)$. No significance difference was found between subjects with intracranial hematoma and normal control group $(p>0.05)$. The levels of TSA in CSF of maligand glioma patients were higher compared to normal control group $(p<0.05)$. The levels of TSA in benign menigioms and metastatic brain tumors were higher compared to normal control group. No significance was found between subjects with intracranal haematoma and normal control group for TSA levels $(p>0.05)$. As showed in Table 2 .

Table 3 shows the plasma level of LSA. TSA and R-SA in subjects with gastric carcinoma were significantly higher than those of normal control group $(p<0.05)$. However, no significant difference was founded in the plasma LSA, TSA and R-SA levels among chronic gastritis, gastrohelcoma and normal control group $(p>0.05)$. Plasma level of LSA, TSA and R-SA in subjects with gastric carcinoma were significantly higher than those of chronic gastritis patients and gastrohelcoma patients $(p<0.05)$.

Table 4 showed that plasma level of LSA, TSA and R-SA in subjects with large intestine carcinoma

Table 1. Compares Level of Total Sialic Acid (TSA) in CFS among Different Groups

\begin{tabular}{lrrc}
\hline Group & $\mathrm{n}$ & $\mathrm{LSA}(\mathrm{u} \mathrm{g} / \mathrm{ml})$ & $\mathrm{P}$ \\
\hline Maligand glioma* & 12 & $9.96 \pm 1.01$ & $<0.05$ \\
Benign menigioms* & 10 & $6.30 \pm 0.76$ & $<0.05$ \\
Metastatic brain tumors & 8 & $10.12 \pm 0.98$ & $<0.05$ \\
Intracranial halmatoma & 8 & $4.25 \pm 0.79$ & $>0.05$ \\
Normal control group & 20 & & \\
\hline
\end{tabular}

Table 2. The Level of TSA in CSF among Different Groups

\begin{tabular}{lrrr}
\hline Group & $\mathrm{n}$ & $\mathrm{LSA}(\mathrm{ug} / \mathrm{ml})$ & $P$-value \\
\hline Maligand glioma* & 12 & $31.3 \pm 5.4$ & $<0.05$ \\
Benign menigioms* & 10 & $26.7 \pm 4.5$ & $<0.05$ \\
Metastatic brain tumors & 8 & $32.2 \pm 4.1$ & $<0.05$ \\
Intracranial halmatoma & 8 & $20.1 \pm 2.9$ & $>0.05$ \\
Normal control group & 20 & $21.5 \pm 3.4$ & \\
\hline
\end{tabular}

Table 3. Comparison of Membrane Glycolipid Levels among Subjects with Gastric Carcinoma, Gastrohelcoma and Chronic Gastritis

\begin{tabular}{|c|c|c|c|c|c|}
\hline Group & $\mathrm{n}$ & $\mathrm{TSA}(\mathrm{ug} / \mathrm{ml})$ & $\mathrm{LSA}(\mathrm{ug} / \mathrm{ml})$ & $\mathrm{R}-\mathrm{SA}(0.1 \mathrm{ug} / \mathrm{ml})$ & $P$-value \\
\hline Gastric carcinoma & 35 & $1122.69 \pm 158.89$ & $346.77 \pm 61.59$ & $26.94 \pm 5.11$ & $<0.05$ \\
\hline Cronic gastritis* & 20 & $928.65 \pm 125.30$ & $263.30 \pm 56.2$ & $21.76 \pm 4.39$ & $<0.05$ \\
\hline Gastrohel-coma* & 20 & $951.76 \pm 112.04$ & $270.12 \pm 53.6$ & $22.56 \pm 5.89$ & $>0.05$ \\
\hline Normal control group & 50 & $934.04 \pm 132.91$ & $266.45 \pm 57.43$ & $22.11 \pm 4.37$ & \\
\hline
\end{tabular}

Table 4. Membrane Gycolipids Level compare of Benign tand Maligant Intestinal Tumors

\begin{tabular}{lcrrrr}
\hline Group & $\mathrm{n}$ & $\mathrm{TSA}(\mathrm{ug} / \mathrm{ml})$ & $\mathrm{LSA}(\mathrm{ug} / \mathrm{ml})$ & $\mathrm{R}-\mathrm{SA}(\mathrm{ug} / \mathrm{ml})$ \\
\hline Intestine maligand tumor* & 30 & $1131.2 \pm 156.3$ & $370.12 \pm 62.51$ & $P$-value & $28.13 \pm 5.35$ \\
Intestine benign tumor & 18 & $950.16 \pm 20.84$ & $272.36 \pm 59.32$ & $22.21 \pm 4.98$ \\
Normal control group & 50 & $934.04 \pm 132.91$ & $266.45 \pm 7.43$ & $22.11 \pm 4.37$ \\
\hline
\end{tabular}

Table 5. Membrane Glycolipid Levels of Intestine Tumor with and without Tumor Metastasis

\begin{tabular}{lcccc}
\hline Group & $\mathrm{n}$ & TSA $(\mathrm{ug} / \mathrm{ml})$ & LSA $(\mathrm{ug} / \mathrm{ml})$ & $\mathrm{R}-\mathrm{SA}(\mathrm{ug} / \mathrm{ml})$ \\
\hline Tumor with metastasis & 12 & $1196.9 \pm 124.36$ & $401.32 \pm 67.58$ & $30.81 \pm 5.03$ \\
No metastasis tumor & 10 & $1057.16 \pm 123.58$ & $385.20 \pm 59$ & $22.12 \pm 4.03$ \\
P-value & & $<0.05$ & $<0.05$ & $<0.05$ \\
\hline
\end{tabular}


were significantly higher than that of subjects with the benign intestine tumor $(p<0.05)$. However, no significant difference was founded between intestine benign tumor and normal control group $(p>0.05)$. The membrane glycolipid levels in subjects with intestine maligand tumor patient was higher than that of subjects with benign tumor $(p<0.05)$.

Table 5 showed the levels of TSA, LSA and R-SA in subjects with intestine tumor metastasis were higher than those without metastasis $(P<0.05)$.

\section{Discussion}

The result of our present study is in accordance with determine of sialic acid content The levels of LSA and TSA in CSF was higher in subjects with intracranial tumor compared to normal control group, the content of TSA and LSA in subjects with malignant glioma was higher than that of subjects with benign meningioms. The present study revealed that plasma LSA, TSA and R-SA levels of gastric carcinoma were significantly higher than those of normal control group. While no significant difference was found in the plasma LSA, TSA and R-SA levels among chronic gastritis, gastrohelcoma and normal control group. The plasma LSA, TSA and R-SA levels of gastric carcinoma patient were significantly higher than those of chronic gestritis patients and gastrohelcoma patients. It was also found that plasma LSA, TSA and R-SA contents were significantly higher in large intestine carcinoma patient than in benign intestine tumor patients, while no significant difference was found between intestine benign tumor and normal control group. The levels of LSA, TSA and R-SA were obviously higher in the patients with metastasis than those without metastasis.

Gangliosides (GLS) is an acidic glycosphingolipid containg $\mathrm{N}$-acetylglycotosamine and ubiquitous in the central nervous system. The term "ganglioside" is meant for the GIS containing sialic acid. The sialic acid (SA) is the generic term given to a family acetylated derivative of neuraminic acid. A main function of host SA is to regulate innate immunity sialic acid occupies the interface between the host and pathogenic microorganisms. Acute phase inflammation has been suggested to associate with infection disease such as periodontal diseases (Pyo et al., 1999; Rusnati et al., 1999).

Sialic aid is a marker of the acute-phase response. Furthemore, it is a prcedictor of several systemic disorders, Cardiovascular disease, rheumatoid arthritis, and diabetes (Iijima et al., 2004; Baenke et al., 2013). Gangliosides are well known for their role in the mediation of several diseascs such as tetanus, botulism and infuenza by acting as cellular receptors for invading pathogens (Stringou et al., 1992; Ariga et al., 2010). While gangliosides of mature brain such as GM1, may be neuroprotective in some circumstances, other ganglioside have long been thought to play an intricate role in the process of cell death in diseases of the central nervous system (Park et al., 2008).

Numerous previous studies reported that higher concentration of plasma or serum gangliosides in cancer patients and acute phase inflammation patients comparing healthy individual, but the mechanism is still unclear
(Basu et al., 2012). The following reason may properly explain the mechanisms of gangliosides levels raised in intracranial tumors and gastrointestinal tumors. firstly, the sudden change of exist chromatin cancer gene due to physical, chemical or biological factor (Byers et al., 2012). Additional, those glycolipid glycosyltransferases activity was remarkable increase lead to membrane glycolipid biosynthesis ability strengthen (Suzuki et al., 2011) .It is has been observed that, tumor cell of neuroectodermal origin may shed their gangliosides into circulation, resulting in higher ganglioside concentration in CSF of intraeranial tumors and plasma of gastrointestinal tumors (Radic et al., 2008).

In conclusion, the membrane glycolipidl biochemistry index LSA and TSA in CSF are sensitive markers for diagnosing intracranial tumors, they have certain significance for identifying malignant tumors from benign ones. In gastrointestinal tumors, the membrane glycolipids biochemistry index plasma LSA, TSA and red blood cell membrane SA may be considered as an auxiliary indicator for diagnosis gastrointestinal malignant tumors. It can be used for distinguishing benign tumors form malignant ones. It can also be regarded as an unspecific indicator for judging tumor metastasis

\section{Acknowledgements}

This work was supported by national natural science foundation of china (No.31301171 and No.30900243) Anhui Province Key Laboratory of active biological macro-molecules (Wannan Medical College).

The CSF sample provide in intracranial tumors, we wish to thank to Dr Chen Yong Quan, the plasma sample provide in gastrointestinal tumors, we wish to thank to Dr Han Zhen of Wannan Medical College First auxiliary hospital.

\section{References}

Ariga T, Yanagisawa M, Wakade C, et al (2010). Ganglioside metabolism in a transgenic mouse model of Alzheimer's disease: expression of Chol-1alpha antigens in the brain. ASN Neuro, $2,44$.

Baenke F, Peck B, Miess H, et al (2013). Hooked on fat: the role of lipid synthesis in cancer metabolism and tumour development. Dis Model Mech, 6, 1353-63.

Banda K, Gregg CJ, Chow R, et al (2012). Metabolism of vertebrate amino sugars with $\mathrm{N}$-glycolyl groups: mechanisms underlying gastrointestinal incorporation of the non-human sialic acid xeno-autoantigen $\mathrm{N}$-glycolylneuraminic acid. $J$ Biol Chem, 287, 28852-64.

Basu S, Ma R, Moskal JR, et al (2012). Ganglioside biosynthesis in developing brains and apoptotic cancer cells: $X$. regulation of glyco-genes involved in GD3 and Sialyl-Lex/a syntheses. Neurochem Res, 37, 1245-55.

Byers DM, Gorbet JC, Irwin LN (2012). Disialogangliosides and TNFalpha alter gene expression for cytokines and chemokines in primary brain cell cultures. Neurochem Res, 37, 214-22.

Gilbert ER, Eby JM, Hammer AM, et al (2013). Positioning ganglioside D3 as an immunotherapeutic target in lymphangioleiomyomatosis. Am J Pathol, 183, 226-34.

Iijima R, Takahashi H, Namme R, et al (2004). Novel biological 
function of sialic acid ( $\mathrm{N}$-acetylneuraminic acid) as a hydrogen peroxide scavenger. FEBS Lett, 561, 163-6.

Lacomba R, Salcedo J, Alegria A, et al (2011). Effect of simulated gastrointestinal digestion on sialic acid and gangliosides present in human milk and infant formulas. $J$ Agric Food Chem, 59, 5755-62.

Mansson JE, Fredman P, Nilsson O, et al (1985). Chemical structure of carcinoma ganglioside antigens defined by monoclonal antibody C-50 and some allied gangliosides of human pancreatic adenocarcinoma. Biochim Biophys Acta, 834, 110-7.

Park JE, Wu DY, Prendes M, et al (2008). Fine specificity of natural killer T cells against GD3 ganglioside and identification of GM3 as an inhibitory natural killer T-cell ligand. Immunology, 123, 145-55.

Pyo H, Joe E, Jung S, et al (1999). Gangliosides activate cultured rat brain microglia. J Biol Chem, 274, 34584-9.

Radic B, Vukelic Z, Bognar SK (2008). Serum gangliosides in patients with brain tumors. Coll Antropol, 32, 171-5.

Rathod SR, Khan F, Kolte AP, et al (2014). Estimation of salivary and serum total sialic Acid levels in periodontal health and disease. J Clin Diagn Res, 8, 19-21.

Rusnati M, Tanghetti E, Urbinati C, et al (1999). Interaction of fibroblast growth factor-2 (FGF-2) with free gangliosides: biochemical characterization and biological consequences in endothelial cell cultures. Mol Biol Cell, 10, 313-27.

Sagawa J, Miyagi T, Tsuiki S (1988). Membrane-associated sialidase of rat liver and its decrease in hepatomas. Jpn $J$ Cancer Res, 79, 69-73.

Schiopu C, Flangea C, Capitan F, et al (2009). Determination of ganglioside composition and structure in human brain hemangioma by chip-based nanoelectrospray ionization tandem mass spectrometry. Anal Bioanal Chem, 395, 2465-77.

Stringou E, Chondros K, Kouvaris J, et al (1992). Serum sialic acid (TSA/LSA) and carcinoembryonic antigen (CEA) levels in cancer patients undergoing radiotherapy. Anticancer Res, 12, 251-5.

Suzuki Y, Yanagisawa M, Ariga T, et al (2011). Histone acetylation-mediated glycosyltransferase gene regulation in mouse brain during development. J Neurochem, 116, 874-80.

Yin X, Xiang T, Li L, et al (2013). DACT1, an antagonist to Wnt/beta-catenin signaling, suppresses tumor cell growth and is frequently silenced in breast cancer. Breast Cancer Res, 15, 23. 